\title{
Theranostics
}

Research Paper

2011; 1:230-239

\section{Photosensitizer-Conjugated Human Serum Albumin Nanoparticles for Eifective Photodynamic Therapy}

\section{Hayoung Jeong ${ }^{1,2 *}$, MyungSook Huh ${ }^{1 *}$, So Jin Lee ${ }^{1}$, Heebeom Koo ${ }^{1}$, Ick Chan Kwon ${ }^{1}$, Seo Young Jeong ${ }^{2}{ }^{凶}$, Kwangmeyung Kim ${ }^{1 凶}$}

1. Biomedical Research Center, Korea Institute of Science and Technology, 39-1 Haweolgog-Dong, Sungbook-Gu, Seoul 136-791, Korea.

2. Department of Life and Nanopharmaceutical Science, Kyung Hee University, 1 Hoegi-dong, Dongdaemun-gu, Seoul, 130-701, Korea.

* These authors contributed equally to this paper.

Corresponding author: Kwangmeyung Kim, PhD, KIST, Biomedical Research Center, Haweolgog-Dong, Sungbook-Gu, Seoul 136-791, Korea. Tel: +82 2958 5916; Fax: +82 2958 5909. E-mail: kim@kist.re.kr. Seo Young Jeong, PhD, Kyung Hee University, Department of Life and Nanopharmaceutical Science, 1 Hoegi-dong, Dongdaemun-gu, Seoul, 130-701, Korea. E-mail: syjeong@khu.ac.kr

(C) Ivyspring International Publisher. This is an open-access article distributed under the terms of the Creative Commons License (http://creativecommons.org/ licenses/by-nc-nd/3.0/). Reproduction is permitted for personal, noncommercial use, provided that the article is in whole, unmodified, and properly cited.

Received: 2011.02.12; Accepted: 2011.03.30; Published: 2011.04.06

\begin{abstract}
Photodynamic therapy (PDT) is an emerging theranostic modality for various cancers and diseases. The focus of this study was the development of tumor-targeting albumin nanoparticles containing photosensitizers for efficient PDT. To produce tumor-targeting albumin nanoparticles, the hydrophobic photosensitizer, chlorin e6 (Ce6), was chemically conjugated to human serum albumin (HSA). The conjugates formed self-assembled nanoparticle structures with an average diameter of $88 \mathrm{~nm}$ under aqueous conditions. As expected, the Ce6-conjugated HSA nanoparticles (Ce6-HSA-NPs) were nontoxic in their native state, but upon illumination with the appropriate wavelength of light, they produced singlet oxygen and damaged target tumor cells in a cell culture system. Importantly, when the nanoparticles were injected through the tail vein into tumor-bearing HT-29 mice, Ce6-HSA-NPs compared with free $\mathrm{Ce} 6$ revealed enhanced tumor-specific biodistribution and successful therapeutic results following laser irradiation. These results suggest that highly tumor-specific albumin nanoparticles have the potential to serve not only as efficient therapeutic agents, but also as photodynamic imaging (PDI) reagents in cancer treatment.
\end{abstract}

Key words: Photodynamic therapy, Human Serum Albumin Nanoparticles

\section{Introduction}

Photodynamic therapy (PDT) is an effective medical tool for treating various cancers by inducing cell apoptosis or necrosis [1-3]. Photodynamic therapy is a theranostic treatment that uses chemical photosensitization and light irradiation at specific wavelengths to eradicate target tumor tissues. In the ab- sence of light, photosensitizers are nontoxic to cells, but when illuminated with specific activating wavelengths, the photosensitizers generate cytotoxic singlet oxygen that destroys cells through the aforementioned mechanisms $[4,5]$. In addition, photosensitizers are able to provide intense fluorescence signals in 
tumor tissues that allow their photodynamic imaging (PDI). Therefore, the selective accumulation of photosensitizers in target tumor tissues can be concurrently employed for both PDI and PDT. However, the use of photosensitizers has been limited in clinical applications because of their poor water solubility and low tumor-targeting efficacy [6].

To overcome these limitations of photosensitizers for PDI and PDT, various nano-sized drug carriers such as polymeric micelles, emulsions, and nanoparticles have been developed to enhance their water solubility and tumor-targeting efficacy [7-10]. Nano-sized drug carriers are known to accumulate at the tumor site by the so-called enhanced permeation and retention (EPR) effect, resulting in efficient passive accumulation in solid tumor tissues [11]. Therefore, various photosensitizer-encapsulated nano-sized carriers could enhance tumor-targeting specificity and therapeutic efficacy in cancer treatment in comparison with free photosensitizers [4, 8, 9]. Recently, these photosensitizer-encapsulated drug carriers have shown great promise for theranostic multifunctional nanoparticles for both the diagnosis and therapeutic treatment of cancers [8].

Human serum albumin (HSA) has attracted recent interest as a theranostic drug carrier due to its abundance, biocompatibility, and versatile roles in imaging and drug delivery $[12,13]$. Moreover, accumulation of albumin at the target tumor tissue provides a rationale for the development of HSA-based drug delivery systems for cancer treatment. In previous studies, HSA-based nanoparticles were made by emulsion formation, desolvation, and coacervation methods [12-15]. In addition, albumin was employed to produce photosensitizer-conjugated drug carriers for cancer treatment [16]. To increase the driving force for nanoparticle formation, specific hydrophobic molecules have been conjugated to native albumin, given that hydrophobic drugs can be successfully loaded into albumin-based nanoparticles [15]. However, there are still a number of problems associated with the development of albumin nanoparticles as drug carriers, including complicated fabrication methods, wide size distribution, instability under physiological conditions, and the unintended burst release of drugs [17].

Herein, chlorin e6 (Ce6)-conjugated HSA nanoparticles (Ce6-HSA-NPs) are introduced as photodynamic drug delivery candidates with biocompatibility and tumor-targeting abilities. To produce tumor-targeting albumin nanoparticles, the hydrophobic photosensitizer Ce6 was directly conjugated to albumin. Chlorin e6 was chosen as an attractive hydrophobic core, providing the driving force for self-assembly. In this paper, the synthesis of Ce6-HSA-NPs is reported, as well as the measurement of the photo-physical properties of the nanoparticles, such as size distribution and the production of singlet oxygen. The cellular uptake and laser-triggered phototoxicity of these nanoparticles were also evaluated in a cell culture system. Finally, the time-dependent biodistribution and the therapeutic efficacy of the nanoparticles in vivo were investigated in HT-29 human colon cancer-bearing mice. The results of this study indicate that the Ce6-HSA-NPs may exhibit prolonged circulation in the blood along with enhanced tumor specificity and improved therapeutic efficacy.

\section{Materials and Methods}

\section{Materials.}

Human serum albumin (HSA, Average MW=67,000), Ce6, N-hydroxysuccinimide (NHS), and 1-ethyl-3-(3-dimethylaminopropyl)-carbodiimide hydrochloride (EDC) were purchased from Sigma (St. Louis, MO). Anhydrous dimethyl sulfoxide (DMSO) and methanol were obtained from Merck (Darmstadt, Germany). All other chemicals and solvents were of analytical grade and were used without further purification. HeLa human cervical cancer cells and HT-29 human colon cancer cells were obtained from the American Type Culture Collection (Rockville, MD).

\section{Preparation of Ce6-HSA-NPs.}

To prepare Ce6-HSA-NPs, Ce6 and HSA were conjugated together in a DMSO/water co-solvent, as described below. (The ratio of DMSO to distilled water in the co-solvent was 1:9.) HSA powder (50 mg) was dissolved in phosphate buffered saline (PBS) $(\mathrm{pH}=7.4,9 \mathrm{ml})$. Three different quantities of Ce6 (2.3, 4.5 , and $14.25 \mathrm{mg}$, corresponding to a mole ratio of $\mathrm{Ce} 6$ to HSA of 5, 10, and 30:1, respectively) were dissolved in DMSO ( $1 \mathrm{ml})$, followed by the addition of 4.5 molar equivalent of NHS and EDC about Ce6. After allowing the HSA and Ce6 solutions to completely dissolve, they were mixed slowly, and the reaction mixture was gently stirred for 1 day. The reaction mixture was dialyzed for 3 days against methanol and distilled water to remove unconjugated Ce6 and DMSO and then lyophilized to produce a light green solid powder containing the Ce6-HSA-NPs. The Ce6-HSA-NPs, which, as noted above, were synthesized with 5,10 , or $30 \mathrm{Ce} 6$ molecules per one molecule of HSA (denoted as Ce6-HSA-NP-5X, Ce6-HSA-NP-10X and Ce6-HSA-NP-30X, respectively), were dissolved in the DMSO/water co-solvent. The solutions were centrifuged three times for $10 \mathrm{~min}$ at 13,200 rpm. Following each centrifugation, the supernatants were 
collected, the pellets were discarded, and the supernatants were again lyophilized.

\section{Characterization of Ce6-HSA-NPs.}

The diameter and size distributions of the Ce6-HSA-NPs were measured with dynamic light scattering (DLS) using a 127-35 laser (Spectra Physics, Mountain View, CA). Ce6-HSA-NPs were dispersed in PBS $\left(1 \mathrm{mg} / \mathrm{ml}, \mathrm{pH} 7.4,37^{\circ} \mathrm{C}\right)$, and the size distribution was measured with a BI-9000AT digital autocorrelator (Brookhaven, NY, USA) operated at $633 \mathrm{~nm}$ and $37^{\circ} \mathrm{C}$. The morphology of the nanoparticles was observed using a transmission electron microscope (TEM) (CM30 electron microscope, Philips, CA) operated at an acceleration voltage of $80 \mathrm{kV}$. Samples (1 $\mathrm{mg} / \mathrm{ml}$ in distilled water) were placed on a 300-mesh copper grid coated with carbon. After drying, each sample was negatively stained using a droplet of 2 $w t \%$ uranyl acetate solution. The Ce6 conjugation efficiency of the Ce6-HSA-NPs was determined using a Lambda UV-Vis 7 spectrophotometer (Perkin-Elmer, CT). A DMSO/water co-solvent was added to the lyophilized nanoparticles $(1 \mathrm{mg} / \mathrm{ml})$ to obtain a clear solution. (The ratio of DMSO to distilled water was 1:9, as noted above.) The concentration of the conjugated $\mathrm{Ce} 6$ was quantified by measuring the absorbance at $405 \mathrm{~nm}$ and comparing the reading to a standard concentration curve of free $\mathrm{Ce} 6$ in the same DMSO/water co-solvent.

\section{Singlet oxygen generation from Ce6-HSA-NPs.}

The capacity of singlet oxygen generation was observed using p-nitroso-N, N'-dimethylaniline (RNO) as a singlet oxygen sensor. The method of bleaching the corresponding endoperoxide of RNO was used to measure the quantum yields of singlet oxygen [18]. Briefly, $100 \mu \mathrm{l}$ of RNO in heavy water $\left(\mathrm{D}_{2} \mathrm{O}, 250 \mu \mathrm{M}\right)$ and $300 \mu \mathrm{l}$ of histidine in $\mathrm{D}_{2} \mathrm{O}(0.03 \mathrm{M})$ were dissolved separately and then mixed together in a quartz cuvette. Free Ce6 $(1 \mu \mathrm{g}, 6.67 \mu \mathrm{M})$ and Ce6-HSA-NPs ( $1 \mu \mathrm{g}$ of Ce6) were dissolved in $700 \mu \mathrm{l}$ of a $\mathrm{D}_{2} \mathrm{O}$ solution containing $1 \%$ DMSO. To measure the singlet oxygen generation, $700 \mu \mathrm{l}$ of each solution was added to the RNO solution. Before measuring the singlet oxygen generation, the solution was bubbled with water-saturated oxygen for $10 \mathrm{~min}$, and the solution was then irradiated with a laser $(671 \mathrm{~nm} 6$ $\left.\mathrm{Jcm}^{-2}\right)$ for $30 \mathrm{~min}$. The optical density at $440 \mathrm{~nm}(\lambda \max$ of RNO) was monitored every 2 min using a spectrophotometer (ISS, Champaign, IL).

\section{Cellular uptake of Ce6-HSA-NPs.}

HeLa cells were cultured with Dulbecco's Modified Eagle's Medium (DMEM) (Gibco, Grand Island, NY) containing 10\% fetal bovine serum (FBS) (Gibco).
To monitor the cellular uptake of Ce6-HSA-NPs, HeLa cells $\left(2 \times 10^{5}\right.$ cells) were seeded onto 6-well plates and allowed to attach for 1 day. The next day, the medium was replaced with $2 \mathrm{ml}$ of serum-free medium, and cells were treated with free Ce6 $(2.5 \mu \mathrm{g} / \mathrm{ml})$ or Ce6-HSA-NPs $(2.5 \mu \mathrm{g} / \mathrm{ml}$ of Ce6) for $1 \mathrm{~h}$. The cells were then washed twice with PBS containing $\mathrm{Ca}^{2+}$ and $\mathrm{Mg}^{2+}$ and stained with DAPI (300 nM) for 5 min. After staining, cells were washed twice with PBS containing $\mathrm{Ca}^{2+}$ and $\mathrm{Mg}^{2+}$ and then fixed with $4 \%$ paraformaldehyde. Using a fluorescence microscope (IX81, Olympus), the cellular uptake and subcellular localization of the Ce6-HSA-NPs were monitored under high resolution. The fluorescence images were observed with a DAPI filter set $(E x=360-370 \mathrm{~nm}$, $E m=420-460 \mathrm{~nm})$ and a Cy5.5 filter set $(E x=640-660$ $\mathrm{nm}, \mathrm{Em}=712-730 \mathrm{~nm}$ ).

\section{Laser-triggered phototoxicity of Ce6-HSA-NPs in a tumor cell culture system.}

To compare the phototoxicity between the free Ce6 and the Ce6-HSA-NPs, HT-29 cells $\left(1 \times 10^{4}\right.$ cells $)$ were seeded onto 96-well cell culture plates and incubated for 1 day. The medium in each well was then replaced with $200 \mu \mathrm{l}$ of serum-free medium containing free Ce6 $(0.5,1,2,4$, or $8 \mu \mathrm{g} / \mathrm{ml})$ or Ce6-HSA-NPs (employing the same concentrations as free Ce6). Control cells received only serum-free medium. The cells were then incubated for $1 \mathrm{~h}$, and then washed twice with PBS before fresh culture medium was added. Following these procedures, the cells were irradiated with an LED lamp (670-690 nm wavelength) for $20 \mathrm{~min}$ and incubated at $37^{\circ} \mathrm{C}$ for $24 \mathrm{~h}$. Cell viability was measured by the MTT colorimetric assay and recorded as the percentage of live cells in the treated samples compared with the percentage of live cells in the untreated control. The assay was performed five times to ensure statistical significance. To visualize dead cells in vitro after PDT, HT-29 cells $\left(2 \times 10^{5}\right.$ cells) were cultured for 2 days in 6-well cell culture plates on cover-slips which had been coated with gelatin. The cells were then irradiated with a He-Ne laser $\left(671 \mathrm{~nm}, 6 \mathrm{~J} / \mathrm{cm}^{2}\right)$ for $2 \mathrm{~min}$ and stained with trypan blue dye for $5 \mathrm{~min}$.

\section{Tumor-targeting efficiency of Ce6-HSA-NPs in HT-29 tumor-bearing mice.}

To evaluate the in vivo biodistribution of free Ce6 and Ce6-HSA-NPs, 5-week-old HT-29 tumor bearing athymic nude mice were prepared (Institute of Medical Science, Tokyo). Subcutaneous tumors were established by intravenous (i.v.) injection of HT-29 cells $\left(5 \times 10^{6}\right.$ cells) into the left side of the back of the mice. When the tumor volume reached approximately 
150-200 $\mathrm{mm}^{3}$, a saline solution of free Ce6 or Ce6-HSA-NPs containing $2.5 \mathrm{mg} / \mathrm{kg}$ of Ce6 was injected through the tail vein into the tumor-bearing mice. After injection, the biodistribution of free Ce6 and Ce6-HSA-NPs within the mice was monitored by using the eXplore Optix system (Advanced Research Technologies Inc., Montreal, Canada). The mice were placed on an animal plate that was heated to $36^{\circ} \mathrm{C}$. Laser power and count time sets were optimized at 6 $\mu \mathrm{W}$ and $0.3 \mathrm{~s}$ per point. The excitation and emission spots were raster-scanned in 1-mm steps over the region of interest to generate emission wavelength scans. A 670-nm pulsed laser diode was used to excite the Ce6 molecules. Long wave fluorescence emission (600-700 nm) was detected using a fast photomultiplier tube (Hamamatsu, Japan) and a time-correlated single photon counting system (Becker and Hickl GmbH, Berlin, Germany).

For determine the blood clearance of free Ce6 and Ce6-HSA-NPs, venous blood samples were collected with heparin coated syringe (31G needle) at each time point $(1,6,12$ and $24 \mathrm{~h})$. Plasma were prepared and measured Ce6 fluorescence intensity with a Cy5.5 filter set using a 12-bit CCD camera (Image Station 4000 MM; Kodak, New Haven, CT) equipped with a special C-mount lens and a long wave emission filter (600 to $700 \mathrm{~nm}$; Omega Optical).

To compare the tissue and tumor accumulation of free Ce6 and Ce6-HSA-NPs, mice were sacrificed after 2 days following i.v. injection of either free Ce6 or Ce6-HSA-NPs (2.5 mg/kg of Ce6), respectively. The excised tissues included the livers, lungs, spleens, kidneys, and hearts, as well as the tumors. Tissues were analyzed by measuring the Ce6 fluorescence intensity with a Cy5.5 filter set using a 12-bit CCD camera (Image Station 4000 MM; Kodak, New Haven, CT) equipped with a special C-mount lens and a long wave emission filter (600 to $700 \mathrm{~nm}$; Omega Optical). A quantification of the in vivo tumor target specificity of free Ce6 and Ce6-HSA-NPs was recorded as total photons per centimeter squared per steradian $\left(\mathrm{p} / \mathrm{s} / \mathrm{cm}^{2} / \mathrm{sr}\right)$ per tumor ( $\mathrm{n}=3$ mice per group).

\section{Therapeutic efficacy of Ce6-HSA-NPs in HT-29} tumor-bearing mice.

As described above, HT-29 tumors were established in athymic nude mice. When the tumor size reached approximately $125 \pm 25 \mathrm{~mm}^{3}$, saline, free Ce6 (2.5 mg/kg of Ce6), and Ce6-HSA-NPs $(2.5 \mathrm{mg} / \mathrm{kg}$ of Ce6) were injected into the mice via the tail vein. At 4 $\mathrm{h}$ and $12 \mathrm{~h}$ post-injection, mice (each group, $\mathrm{n}=3$ ) were irradiated (two times, $30 \mathrm{~min}$ each time) at the tumor site with a laser $\left(671 \mathrm{~nm}, 6 \mathrm{~J} / \mathrm{cm}^{2}\right)$. The therapeutic efficacy of the treatments was next monitored by measuring the tumor volumes calculated as $1 / 2 \times$ (length $\times$ width $^{2}$ ) with a caliper for 25 days. In addition, at 10 days after laser irradiation, tumor tissues were isolated from the mice, fixed with $4 \%$ paraformaldehyde, and embedded in paraffin. The sliced tumor tissues $(6 \mu \mathrm{m})$ were stained with hematoxylin and eosin (H\&E) to evaluate the induction of necrosis or apoptosis.

\section{Results \& Discussion}

In the present study, tumor-targeting albumin-based nanoparticles were prepared as an efficient carrier of the photosensitizer, Ce6. The valuable properties of the Ce6-carrying nanoparticles, such as self-assembly, particle stability, singlet oxygen generation, cellular uptake, and in vitro phototoxicity, were precisely evaluated. Based on these data, the particles were ultimately subjected to in vivo PDI and PDT in a tumor-bearing mouse model.

First, Ce6 was conjugated to the amine groups of the lysine residues within HSA so as to form self-assembling nanoparticles for PDT (Figure 1A). To optimize the content of Ce6 within the Ce6-HSA-NPs for evaluation of in vivo stability and high tumor-targeting ability, variable amounts of Ce6 (5:1, 10:1, 30:1 mole ratio per HSA) were used in the conjugation reaction. Carboxylic groups of Ce6 were activated by EDC/NHS, and amide bonds were formed with the amine residues of HSA through an overnight reaction. To determine the optimized Ce6 conjugation ratio, the amounts of grafted Ce6 were measured by the UV spectra method. As shown in Table 1, Ce6-HSA-5X, Ce6-HSA-10X, and Ce6-HSA-30X demonstrated $59.1 \%, 76.4 \%$ and $38.6 \%$ grafting efficiency, respectively. Residual Ce6s were significantly removed by long-term dialysis with DMSO/water co-solvent. The grafting amount of Ce6 did not increase more than approximately 14 molecules to one albumin molecule. The fact that the grafting efficiency of Ce6-HSA-30X was decreased was likely due to the fact that the reactivity of some of the amines within HSA was limited due to stearic hindrance in the albumin molecule [19]. Therefore, Ce6-HSA-10X was used for all further studies.

Table I. Feed ratio and grafting efficiency of Ce6-HSA-NPs.

\begin{tabular}{lll}
\hline Sample & Grafting amount & Grafting efficiency (\%) \\
\hline Ce6-HSA-NP-5X & 8.76 & 59.1 \\
Ce6-HSA-NP-10X & 14.36 & 76.4 \\
Ce6-HSA-NP-30X & 14.44 & 38.6 \\
\hline
\end{tabular}

aThis number denotes the molar feed ratio of Ce6 to one HSA molecule. 
Under aqueous conditions, the resulting conjugates formed stable self-assembled nanoparticles by hydrophobic interactions between Ce6 molecules. The diameter and size distribution of these Ce6-HSA-NPs dispersed in PBS ( $\mathrm{pH} 7.4,37^{\circ} \mathrm{C}$ ) was measured using DLS. The nanoparticles were about $80-100 \mathrm{~nm}$ in diameter with a stable size distribution and a lack of aggregation, and showed good solubility in contrast to hydrophobic free Ce6 molecules (Figure 1B). The average diameter and size distribution of the nanoparticles was maintained for 7 days (data not shown). TEM images also confirmed their spherical shape, nano-size, and narrow size distribution (Figure 1C). We think the different size of Ce6-HSA-NPs in be- tween DLS data and TEM image was originated from the increase of hydrodynamic volume of nanoparticles in aqueous condition. TEM image was obtained with dried samples, but DLS data was determined in aqueous condition. This nano-size distribution is about 10 -fold larger than the native albumin molecule and is expected to demonstrate increased tumor accumulation, resulting from both longer periods in the blood and the EPR effect, as discussed above and in previous studies $[11,20,21]$. Furthermore, the nature of this drug conjugation could minimize the unintended burst release and the consequential loss of photosensitizers during blood circulation.
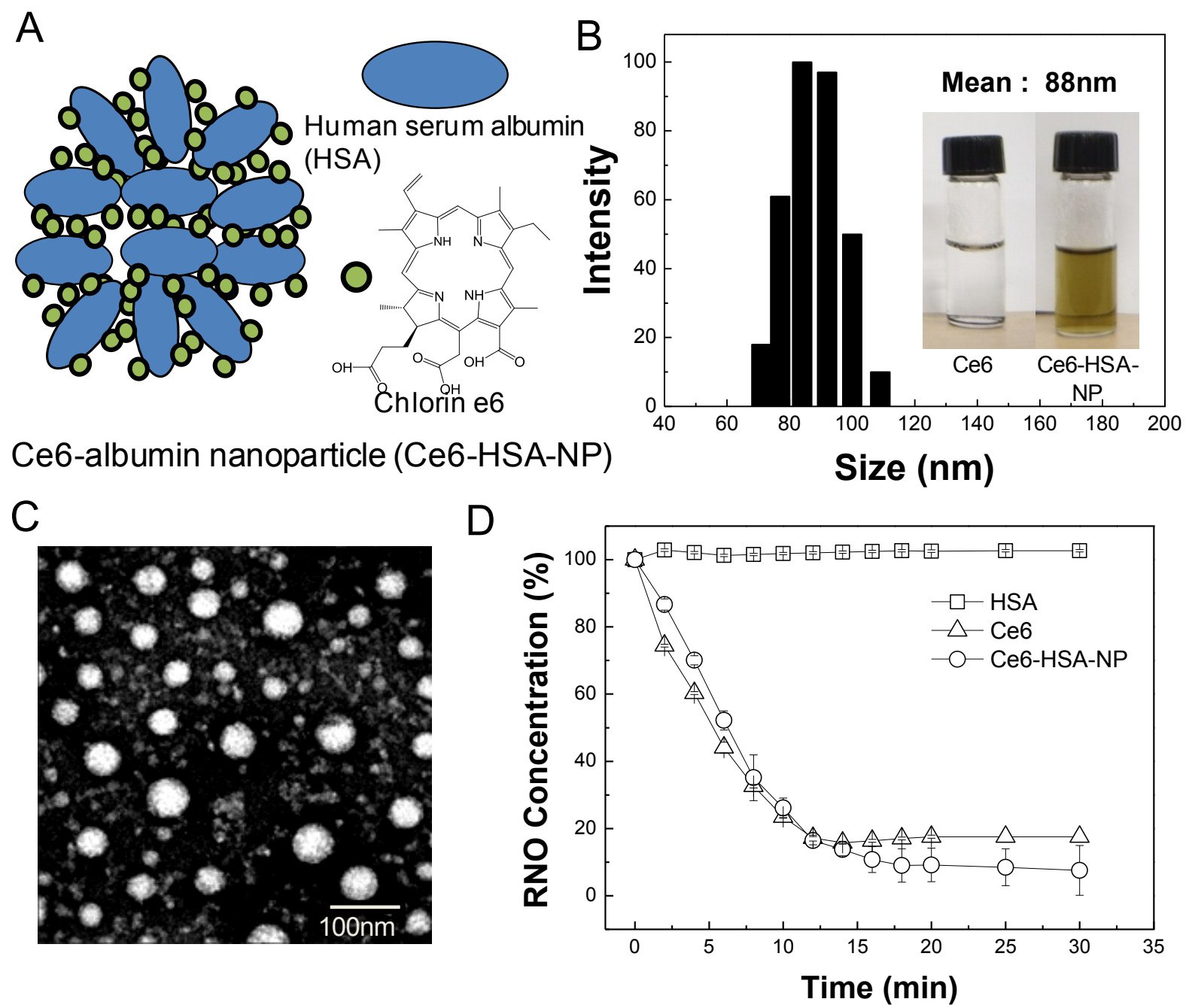

Figure I. Synthesis and characterization of $\mathrm{Ce} 6$ conjugated-human serum albumin nanoparticles (Ce6-HSA-NPs). (A) Illustration of self-assembled Ce6-HSA-NPs, (B) Size distribution of Ce6-HSA-NPs (inset images are vials containing PBS solutions of free $\mathrm{Ce} 6$ and Ce6-HSA-NPs). (C) TEM image of Ce6-HSA-NPs (D) Singlet oxygen generation of free Ce6 and Ce6-HSA-NPs according to irradiation time. 
The singlet oxygen generation of Ce6-HSA-NPs was measured using RNO as a singlet oxygen sensor and a UV spectrometer (Figure 1D). HSA dissolved in $1 \%$ DMSO did not cause any reactive oxygen production after illumination by laser treatment. However, following illumination of Ce6, optical density decreased rapidly, indicating the generation of singlet oxygen. In the case of Ce6-HSA-NPs, the singlet oxygen generation in the $1 \%$ DMSO solution was similar to that produced by illuminated free $\mathrm{Ce} 6$, demonstrating that the therapeutic activities of the Ce6 molecules in Ce6-HSA-NPs were not changed after conjugation to HSA molecules.

Next, cellular uptake of free Ce6 and Ce6-HSA-NPs by HeLa cells was imaged using confocal fluorescence microscopy (Figure 2A). The chlorin ring structure of $\mathrm{Ce} 6$ generates a strong fluores- cence signal, enabling confirmation of the uptake and intracellular localization of the molecule through deep red fluorescence emission $(E x=640-660 \mathrm{~nm}$, $\mathrm{Em}=712-730 \mathrm{~nm})$. In comparison with free Ce6, Ce6-HSA-NPs showed similar cellular uptake, and the amount of Ce6 in the cytosol was not decreased following conjugation to HSA. To evaluate cellular localization of Ce6-HSA-NPs, the nucleus was stained with DAPI, and the localization of Ce6 was observed in high resolution images after uptake of Ce6-HSA-NPs. As shown in Figure 2B, the red fluorescence of the Ce6-HSA-NPs was not observed in the nucleus (stained blue using DAPI) and was only localized in the cytoplasm. Because the main target of photosensitizers is mitochondrial, this result suggests the possibility of effective tumor cell death resulting from PDT with Ce6-HSA-NPs.
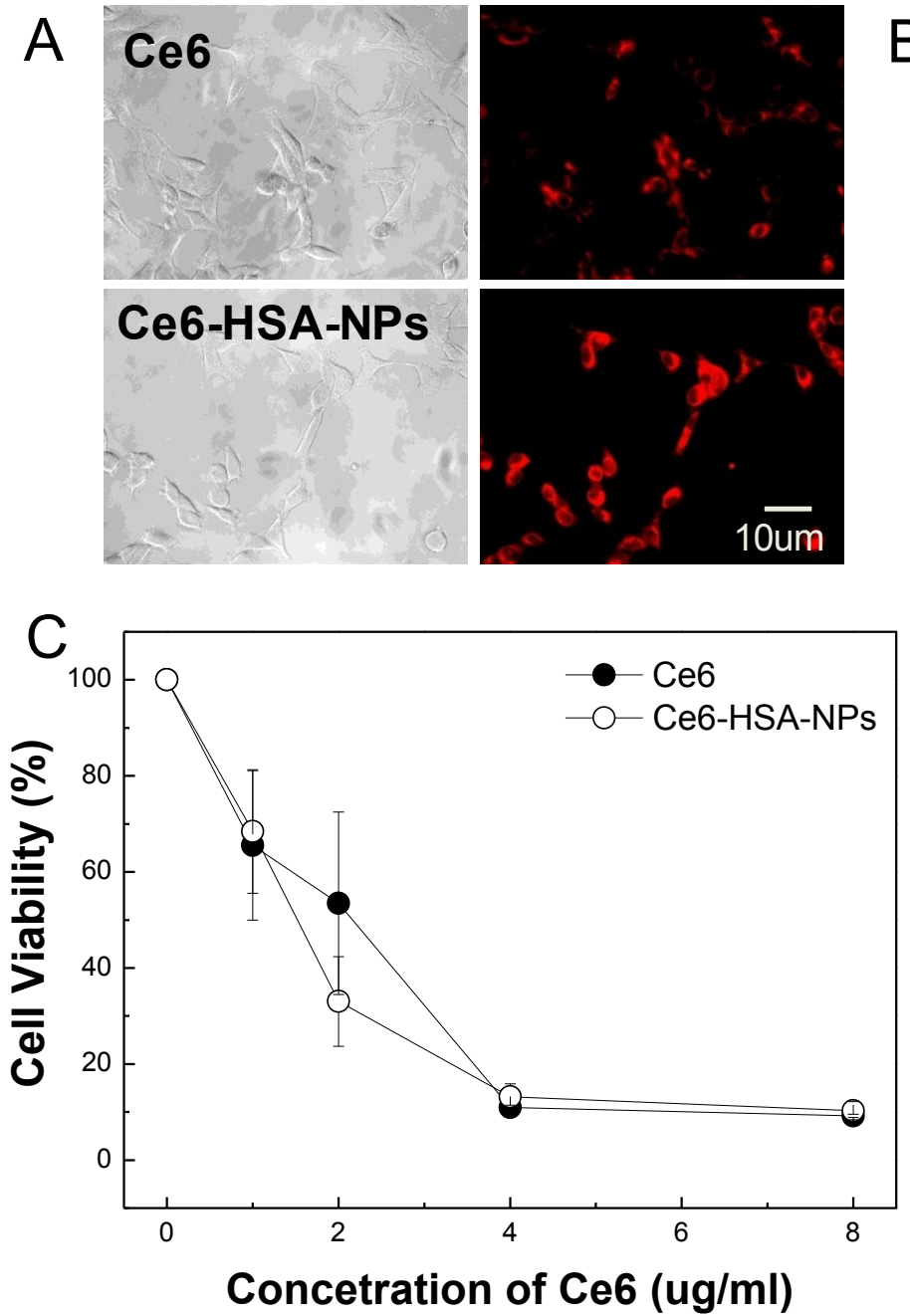
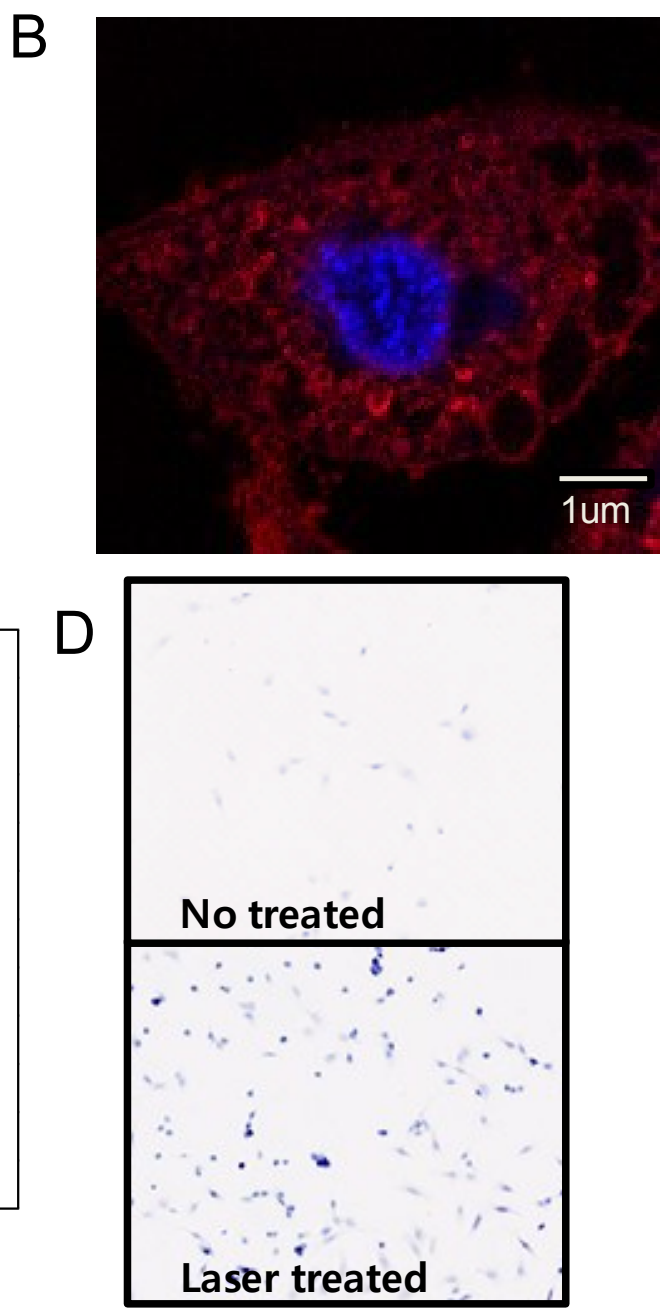

Figure 2. Cellular uptake and in vitro phototoxicity of Ce6-HSA-NPs. (A) Cellular uptake of free Ce6 and Ce6-HSA-NPs into HeLa cells. (B) Intracellular localization of Ce6-HSA-NPs with blue DAPI staining. (C) MTT assay in HeLa cells treated with free Ce6/Ce6-HSA-NPs and 20 min of LED lamp irradiation. (D) Trypan blue staining images of HeLa cells irradiated with laser for 30 min after treating Ce6-HSA-NPs. 
To evaluate the phototoxicity in vitro of Ce6-HSA-NPs, HT-29 cells were incubated with free Ce6 and Ce6-HSA-NPs for $1 \mathrm{~h}$ and then washed with PBS. Next, cells were irradiated using a laser for 20 min, and cell viability was determined using the MTT assay (Figure 2C). No cytotoxicity was observed in the case of HSA regardless of irradiation, and almost all of the cells were viable in the case of both free Ce6 and Ce6-HSA-NPs in the absence of irradiation (data not shown). However, following irradiation with the appropriate wavelength of light, cell viability dramatically decreased according to the concentration of Ce6 in HT-29 cells treated with free Ce6 and Ce6-HSA-NPs. Laser-triggered phototoxicity of Ce6-HSA-NPs was also observed using the trypan blue viability assay (Figure 2D). After $1 \mathrm{~min}$ of irradiation, blue spots were observed only in the laser-treated regions, which demonstrated that most of irradiated cells were severely damaged or destroyed. In the same slide, cells outside the laser-irradiated area were mostly viable (data not shown). This result demonstrated similar in vitro phototoxicity of free Ce6 and Ce6-HSA-NPs and was in accordance with singlet oxygen generation and cellular uptake data.

Based on the intrinsic fluorescence of $\mathrm{Ce} 6$, the tumor-targeting ability of Ce6-HSA-NPs in HT-29 tumor-bearing mice was evaluated in a noninvasive manner. After i.v. injection of free Ce6 $(2.5 \mathrm{mg} / \mathrm{kg})$ and Ce6-HSA-NPs $(2.5 \mathrm{mg} / \mathrm{kg}$ of Ce6) into the tail vein, the time-dependent biodistribution of free $\mathrm{Ce} 6$ and Ce6-HSA-NPs was monitored using an in vivo optical imaging system (Figure 3A). When free Ce6 was injected into the mice, a strong fluorescent signal was observed primarily in liver tissues, and the signal in the whole body was only maintained for up to $6 \mathrm{~h}$. This result indicated that injected free Ce6 was eliminated rapidly, providing low accumulation in tumor tissues. However, in case of Ce6-HSA-NP-treated mice, an enhanced fluorescent signal throughout the whole body persisted for nearly $12 \mathrm{~h}$, and the signal at the tumor site was stronger than that observed in other tissues. Hence, the prolonged circulation time of Ce6-HSA-NPs in the blood was mainly attributed to the high accumulation of the nanoparticles in the tumor tissue. The time-dependent accumulation of Ce6-HSA-NPs in tumor tissue is clearly shown in Figure 3B, and the fluorescent signal was far stronger than that observed in the free Ce6-treated mice. Importantly, even after $1 \mathrm{~h}$ post-injection of Ce6-HSA-NPs, subcutaneous tumors were detected against the surrounding background tissue, indicating the possible use of nanoparticles for PDI, as mentioned above. The fluorescent signal exhibited its maximum intensity at $12 \mathrm{~h}$ post-injection and gradually decreased for 2 days. In addition, the total photon counts from Ce6-HSA-NPs in the tumor tissue were about 13.7-fold higher compared with that of free Ce6 (Figure 3C). To investigate the blood circulation dynamics of Ce6-HSA-NPs, we monitored and compared free $\mathrm{Ce} 6$ or Ce6-HSA-NPs blood circulation (Figure 3D). Ce6-HSA-NPs showed prolonged and slow clearance kinetics within blood until $24 \mathrm{~h}$, while free Ce6 particles rapidly decreased its' fluorescent intensity, this was accordance with tumor accumulation result. One hour later, free $\mathrm{Ce} 6$ was hard to detect from the vein, but Ce6-HSA-NPs could circulate more than $12 \mathrm{~h}$. It represented significant prolonged blood circulation effect of conjugated nanoparticle form. Upon ex vivo evaluation of excised tissues at $48 \mathrm{~h}$ post-injection, Ce6-HSA-NP-treated mice showed a strong fluorescent intensity in the tumor tissue, but unfortunately, the fluorescent intensity in the liver tissue was even stronger (Figure 3E). This indicated that although a large number of Ce6-HSA-NPs accumulated in tumor tissues, the nanoparticles also accumulated in the liver. This result may be explained by the fact that Kupffer cells in the liver play a role to uptake and degrade proteins, including albumin. However, this may not be a critical problem in case of PDT, because the light source cannot penetrate inside liver tissue.

To evaluate the therapeutic efficacy of Ce6-HSA-NPs in HT-29 tumor-bearing mice, HT-29 tumors were established in athymic nude mice as described above. Then, saline, free Ce6 $(2.5 \mathrm{mg} / \mathrm{kg}$ of Ce6), and Ce6-HSA-NPs (2.5 mg/kg of Ce6) were injected into the mice via the tail vein. Mice were irradiated at the tumor site (two times; 30 min each time) with a He-Ne laser at $4 \mathrm{~h}$ and $12 \mathrm{~h}$ post-injection. The appropriate time for laser irradiation was optimized through several pre-test. At 2 days post-injection, Ce6-HSA-NP-treated mice demonstrated a severe tumor necrosis at the site of the laser irradiation. These significant therapeutic effects were not observed in the free Ce6-treated group (Figure 4A). Seven days later, it was apparent that the necrotic scar tissue was healing and that normal tissue had begun to regenerate in the Ce6-HSA-NP-treated mice. The therapeutic efficacy of each treatment was monitored by evaluating the tumor volumes for 25 days (Figure 4B). 

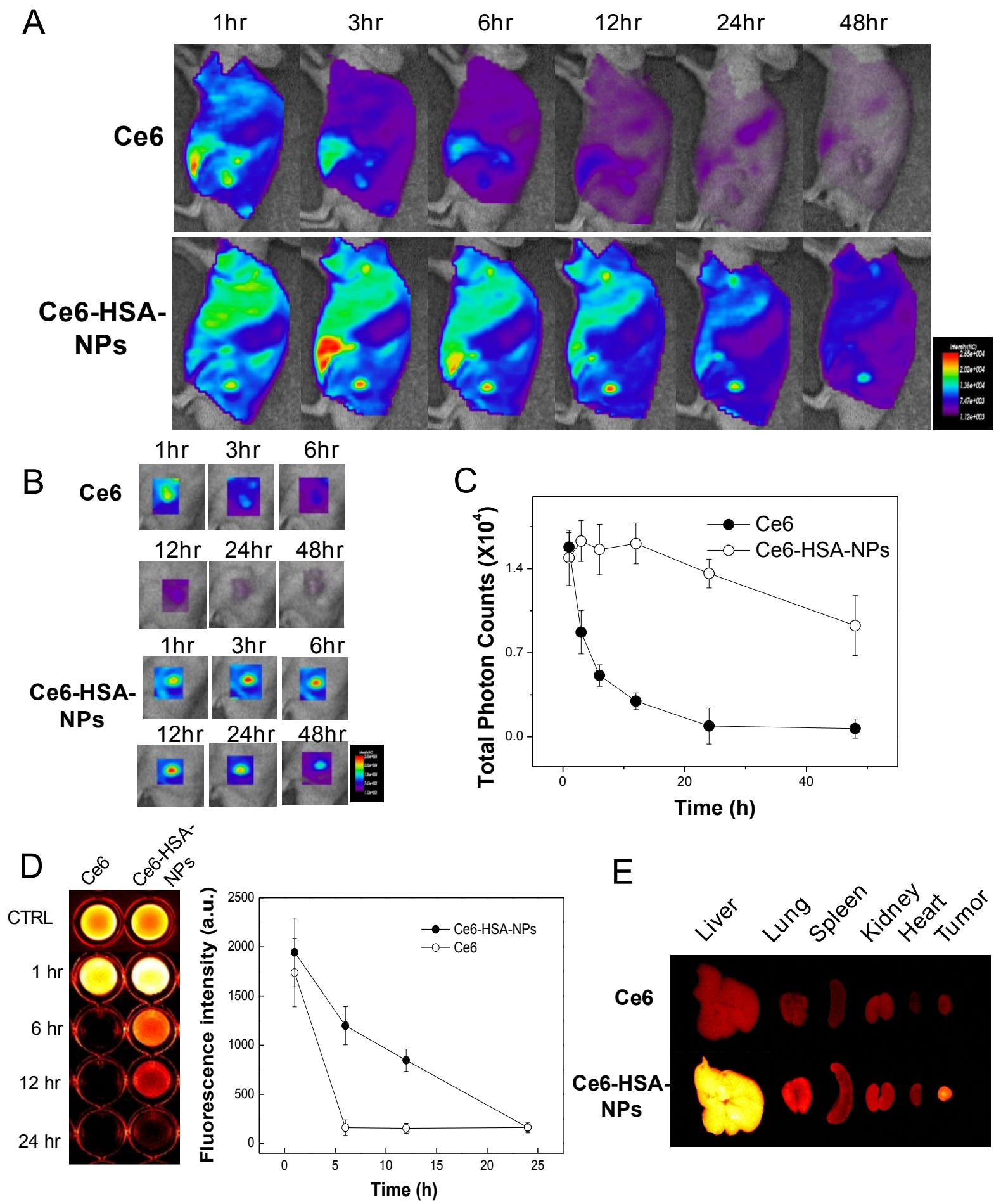

Figure 3. In vivo photodynamic imaging with Ce6-HSA-NPs in tumor-bearing mouse model. (A) in vivo time-dependant whole body imaging of athymic nude mice bearing HT-29 tumors after i.v. injection of free Ce6 and Ce6-HSA-NPs (5mg/kg of Ce6). (B) In vivo tumor target specificity imaging of (A). (C) Quantification of fluorescence signals in tumor site of (A) (total photon counts per centimeter squared per steradian ( $\mathrm{p} / \mathrm{s} / \mathrm{cm}^{2} / \mathrm{sr}$ ) per each tumor). (D) Blood fluorescence of free Ce6 and Ce6-HSA-NP injected HT-29 tumor-bearing mice and the quantification of fluorescent intensity $(n=3)$. (E) Ex vivo images of normal organs (liver, lung, spleen, kidney and heart) and tumors excised at 2 days post-injection of free Ce6 and Ce6-HSA-NPs. 
A

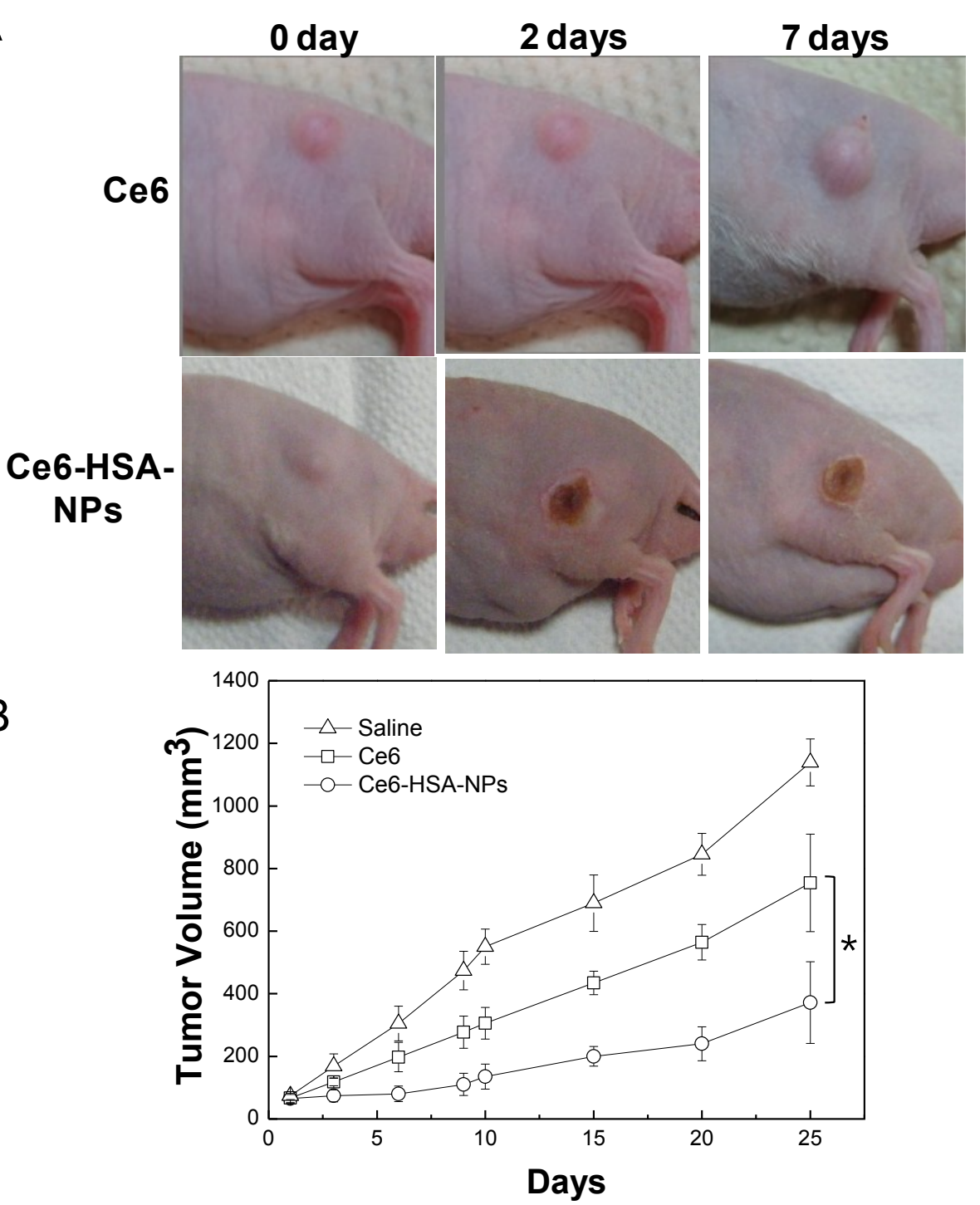

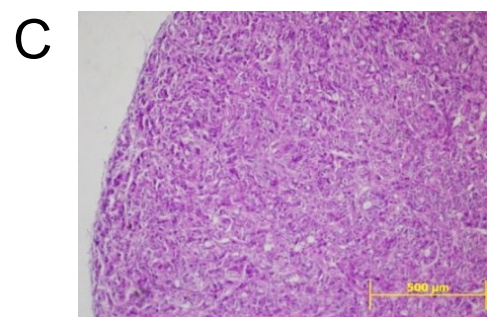

saline

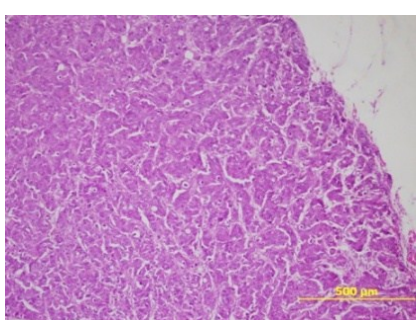

Ce6

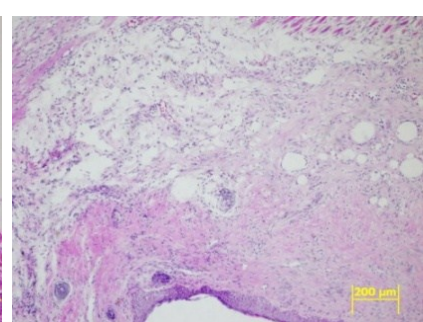

Ce6-HSANPs

Figure 4. In vivo photodynamic therapy with Ce6-HSA-NPs in tumor-bearing mouse model. (A) Resulting images of In vivo photodynamic therapy after i.v. injection of free Ce6 and Ce6-HSA-NPs $(2.5 \mathrm{mg} / \mathrm{kg}$ of Ce6) and 30 min irradiation at tumor site with a laser $\left(67 \mathrm{Inm}, 6 \mathrm{Jcm}^{-2}\right)$ after $4 \mathrm{hr}$ and $12 \mathrm{hr}$ post-injection. (B) Tumor growth data of (A) measured for 25 days. Data represent mean \pm s.e. (The paired Student's test was used for statistical analysis and * denotes significant diffrerence defined as $P<0.05$.) (C) H\&E staining of tumor tissue of $(A)$ at 10 days after treatment. Original magnification is $x 100$. 
Free Ce6-treated mice showed a reduced tumor volume of about $34 \%$ compared with saline-treated mice. Ce6-HSA-NP-treated mice demonstrated a significantly greater tumor reduction of $70 \%$ due to the high tumor-targeting efficacy of the nanoparticles. At 10 days after laser irradiation, the therapeutic result was further evaluated by histological tissue staining with H\&E to confirm the tissue damage (Figure 4C). Saline-injected mice showed tumor cells with rare apoptosis or necrosis. However, in mice treated with Ce6-HSA-NPs, most of the tumor tissue was severely destroyed by irradiation, making it difficult to locate the tumor lesion. Instead, fibrotic tissue was observed behind the epidermis. By contrast, incomplete tumor destruction was observed in mice treated with free Ce6 and irradiation.

In summary, we have developed novel biocompatible tumor-targeting nanoparticles for efficient PDT of cancers. The nanoparticles were formed by self-assembly of photosensitizer-conjugated HSAs. The therapeutic activity of Ce6 molecules in these Ce6-HSA-NPs was not decreased after conjugation, and the Ce6 generated cytotoxic singlet oxygen upon irradiation. Ce6-HSA-NPs exerted higher tumor specificity and therapeutic efficacy compared with free Ce6 following irradiation and singlet oxygen generation. The high accumulation in tumor tissue and efficient therapy with Ce6-HSA-NPs was mainly caused by prolonged circulation in the blood in addition to the EPR effect for the production of stable nano-structures $[20,21]$. These results demonstrate the promising potential of Ce6-HSA-NPs as effective tumor-targeting carriers for PDT of various cancers.

\section{Acknowledgements}

This work was financially supported by the Real-Time Molecular Imaging Project, the Global Research Laboratory Project, the Fusion Technology Project (2009-0081876) of MEST, and the Intramural Research Program of KIST.

\section{Conflict of Interest}

The authors have declared that no conflict of interest exists.

\section{References}

1. Triesscheijn M, Baas P, Schellens JH, Stewart FA. Photodynamic therapy in oncology. Oncologist. 2006;11:1034-44.

2. Dougherty TJ, Gomer CJ, Henderson BW, Jori G, Kessel D, Korbelik M, et al. Photodynamic therapy. J Natl Cancer Inst. 1998;90:889-905.

3. Brown SB, Brown EA, Walker I. The present and future role of photodynamic therapy in cancer treatment. Lancet Oncol. 2004;5:497-508.
4. Bechet D, Couleaud P, Frochot C, Viriot ML, Guillemin F, Barberi-Heyob M. Nanoparticles as vehicles for delivery of photodynamic therapy agents. Trends Biotechnol. 2008;26:612-21.

5. Ackroyd R, Kelty C, Brown N, Reed M. The history of photodetection and photodynamic therapy. Photochem Photobiol. 2001;74:656-69.

6. Chen K, Preuss A, Hackbarth S, Wacker M, Langer K, Roder B. Novel photosensitizer-protein nanoparticles for photodynamic therapy: photophysical characterization and in vitro investigations. J Photochem Photobiol B. 2009;96:66-74.

7. Cheng Y, A CS, Meyers JD, Panagopoulos I, Fei B, Burda C. Highly efficient drug delivery with gold nanoparticle vectors for in vivo photodynamic therapy of cancer. J Am Chem Soc. 2008;130:10643-7.

8. Koo H, Lee H, Lee S, Min KH, Kim MS, Lee DS, et al. In vivo tumor diagnosis and photodynamic therapy via tumoral pH-responsive polymeric micelles. Chem Commun. 2010;46:5668-70.

9. Lee SJ, Park K, Oh YK, Kwon SH, Her S, Kim IS, et al. Tumor specificity and therapeutic efficacy of photosensitizer-encapsulated glycol chitosan-based nanoparticles in tumor-bearing mice. Biomaterials. 2009;30:2929-39.

10. Lowe KC, Akande SL, Bonnett R, White RD, Berenbaum MC. Protective effects of a novel perfluorochemical emulsion in photodynamic therapy. Biomater Artif Cells Immobilization Biotechnol. 1992;20:925-7.

11. Maeda H. Tumor-Selective Delivery of Macromolecular Drugs via the EPR Effect: Background and Future Prospects. Bioconj Chem. 2010;21:797-802.

12. Muller BG, Leuenberger $H$, Kissel T. Albumin nanospheres as carriers for passive drug targeting: An optimized manufacturing technique. Pharm Res. 1996;13:32-7.

13. Gallo JM, Hung CT, Perrier DG. Analysis of Albumin Microsphere Preparation. Int J Pharm. 1984;22:63-74.

14. Weber C, Kreuter J, Langer K. Desolvation process and surface characteristics of HSA-nanoparticles. Int J Pharm. 2000;196:197-200.

15. Gong J, Huo M, Zhou J, Zhang Y, Peng X, Yu D, et al. Synthesis, characterization, drug-loading capacity and safety of novel octyl modified serum albumin micelles. Int $\mathrm{J}$ Pharm. 2009;376:161-8.

16. Wacker M, Chen K, Preuss A, Possemeyer K, Roeder B, Langer K. Photosensitizer loaded HSA nanoparticles. I: Preparation and photophysical properties. Int J Pharm. 2010;393:254-63.

17. Kratz F. Albumin as a drug carrier: Design of prodrugs, drug conjugates and nanoparticles. J Controlled Release. 2008;132:171-83.

18. Kraljić I, Mohsni SE. A NEW METHOD FOR THE DETECTION OF SINGLET OXYGEN IN AQUEOUS SOLUTIONS. US: Blackwell Publishing Ltd. 1978: 577-81.

19. Goldfarb AR. Heterogeneity of Amino Groups in Proteins. I. Human Serum Albumin*. Biochemistry (Mosc). 2002;5:2574-8.

20. Fox ME, Szoka FC, Fréchet JMJ. Soluble Polymer Carriers for the Treatment of Cancer: The Importance of Molecular Architecture. Acc Chem Res. 2009;42:1141-51.

21. Phillips MA, Gran ML, Peppas NA. Targeted nanodelivery of drugs and diagnostics. Nano Today. 2010;5:143-59. 